\title{
Systemic immune-inflammation index predicts prognosis of bladder cancer patients after radical cystectomy
}

\author{
Wentao Zhang ${ }^{1 \#}$, Ruiliang Wang ${ }^{1 \#}$, Wenchao $\mathrm{Ma}^{1,2 \#}$, Yuan $\mathrm{Wu}^{1,2}$, Niraj Maskey ${ }^{1}$, Yadong Guo ${ }^{1}$, Ji Liu ${ }^{1}$, \\ Shiyu Mao ${ }^{1}$, Junfeng Zhang ${ }^{1}$, Xudong Yao ${ }^{1,2}$, Yongzhen Liu ${ }^{1}$ \\ ${ }^{1}$ Department of Urology, Shanghai Tenth People's Hospital, Tongji University, Shanghai 200072, China; ${ }^{2}$ Anhui Medical University, Hefei \\ 230032, China \\ Contributions: (I) Conception and design: X Yao, Y Liu; (II) Administrative support: X Yao; (III) Provision of study materials or patients: W Zhang, \\ R Wang, W Ma; (IV) Collection and assembly of data: Y Wu, J Liu, Y Guo, N Maskey; (V) Data analysis and interpretation: S Mao, J Zhang; (VI) \\ Manuscript writing: All authors; (VII) Final approval of manuscript: All authors. \\ \#These authors contributed equally to this work. \\ Correspondence to: Yongzhen Liu; Xudong Yao. Professor, Department of Urology, Shanghai Tenth People's Hospital, Tongji University, Shanghai \\ 200072, China. Email: 212929001@qq.com; yaoxudong1967@163.com.
}

\begin{abstract}
Background: The systemic immune-inflammation index (SII) has been used as a prognostic marker for several cancer types, but there is no in-depth study in bladder cancer. This study evaluated the potential utility of the SII as a prognostic factor in patients with bladder cancer after radical cystectomy.

Methods: A retrospective analysis of 209 patients with bladder cancer who had undergone radical cystectomy and were randomized into primary $(\mathrm{N}=139)$ and validation $(\mathrm{N}=70)$ cohorts was conducted. The overall survival (OS) was calculated using the Kaplan-Meier survival curves. The prognostic value of the SII in primary and validation cohorts were analyzed by using the Cox regression model. A SII-based nomogram for bladder cancer was produced in R software.
\end{abstract}

Results: A high SII (>507) was associated with poor prognosis in bladder cancer patients. Univariate and multivariate analyses revealed that the SII was an independent predictor for OS. The SII emerged as an independent prognostic factor that provided more accurate prognostic prediction than neutrophillymphocyte ratio (NLR), platelet-lymphocyte ratio (PLR), and C-reactive protein/albumin ratio (CAR), in the primary and validation cohorts. The nomogram had better accuracy and discrimination than tumor, lymph node, metastasis (TNM) classification. The concordance index values of nomogram were 0.82 for the primary cohort and 0.784 for the validation cohort.

Conclusions: The SII can serve as an independent predictor of OS in patients who have undergone radical cystectomy for bladder cancer, and was found to be a better predictor of prognosis than NLR, PLR, and CAR. The nomogram is a reliable model for predicting postoperative OS of patients after radical cystectomy.

Keywords: Bladder cancer; systemic immune-inflammation index (SII); prognosis; immunity

Submitted Jun 20, 2019. Accepted for publication Aug 21, 2019.

doi: $10.21037 /$ atm.2019.09.02

View this article at: http://dx.doi.org/10.21037/atm.2019.09.02

\section{Introduction}

Bladder cancer is the most common malignancy of the urinary system and a serious threat to human health. Globally, it is the fourth and eleventh most common primary cancer among men and women, respectively. Bladder cancer represents the eighth most prevalent cause of male cancer mortality (1), and the first most prevalent urinary system malignancy among Chinese men (2). Most cases $(70-75 \%)$ are diagnosed during a non-muscle-invasive bladder cancer (NMIBC) stage, which is treated mainly by transurethral resection of bladder tumor (TURBT) followed by intravesical instillation chemotherapy, intravesical 
bacillus Calmette-Guérin (BCG) immunotherapy or combination therapy (3).

Approximately $50-70 \%$ of patients with NMIBC will recur within 5 years. In addition, $10-15 \%$ of NMIBC patients progress to muscle invasive bladder cancer (MIBC). MIBC, which is present in about a quarter of new bladder cancer patients, has a 5-year tumor-specific overall survival (OS) rate of only about 50\% (4). High-grade T1 bladder cancer is more likely to progress from NMIBC to MIBC (5). The most effective treatment for MIBC is radical cystectomy (6). Although the bladder is completely removed in radical cystectomy, patients still face a serious risk of cancer recurrence (7). Moreover, there are currently no reliable biomarkers to predict the prognosis of bladder cancer patients with MIBC.

The identification and evaluation of biomarkers are essential for patient risk stratification, treatment planning, and long-term bladder cancer outcomes. In recent years, researchers have found that immune response cells (neutrophils, monocytes, and lymphocytes) and platelets and their associated signaling pathways are important components of the tumor microenvironment and can influence tumor progression and metastasis (8). During inflammatory responses, levels of neutrophils, platelets, and acute phase proteins, such as C-reactive protein and albumin, are altered, providing a several easily measured parameters. Many of these inflammatory factors represent potential biomarkers of cancer prognosis. In particular, the inflammatory cell markers neutrophil-lymphocyte ratio (NLR), platelet-lymphocyte ratio (PLR), and C-reactive protein/albumin ratio (CAR) have been used to evaluate cancer recurrence and survival (9-11). The systemic immune-inflammation index (SII), which is based on peripheral lymphocyte, neutrophil, and platelet counts, has come into use recently as an assessment of the preoperative balance of inflammatory factors and immune status, to enable better predictions of prognosis.

Although the prognostic value of the SII has been confirmed for gastric cancer, colorectal cancer, esophageal cancer, and other tumors (12-14), its value for bladder cancer prognostication has not been established. Therefore, the aim of this study was to explore the clinical significance of the SII as a predictor of OS in patients treated for bladder cancer with radical cystectomy.

\section{Methods}

The case files of 240 patients who underwent radical cystectomy at the Shanghai Tenth People's Hospital (Shanghai, China) from January 2005 to February 2019 were collected retrospectively. The inclusion criteria were as follows: (I) histopathological diagnosis of bladder cancer after TURBT, including MIBC and high-grade T1 bladder cancer; (II) complete pathology, laboratory, and followup data. Patients receiving neoadjuvant chemotherapy and patients with an active infection or inflammatory disease within 1 month preoperatively were excluded. Thirty-one patients were lost to follow-up, so a total of 209 patients were ultimately eligible for and included in the study and randomized into a primary cohort $(\mathrm{N}=139)$ and validation cohort $(\mathrm{N}=70)$.

The following demographic, clinical, and pathology data were collected from patient medical records: age, gender, body mass index (BMI), tumor size, tumor history, laboratory test results, (neutrophil count, lymphocyte count, C-reactive protein), computed tomography imaging findings, and surgery method used. Pathologists assessed the tumor grade and stage according to the $2004 \mathrm{WHO}$ classification system and tumor, lymph node, metastasis $(\mathrm{TNM})$ rubric. SII was calculated as platelet count $\times$ neutrophil count/lymphocyte count. CAR, NLR, and PLR were calculated as C-reactive protein/albumin, neutrophil number/lymphocyte number, and platelet count/lymphocyte count, respectively.

The patients were followed up by two experienced nonsurgeon physician assistants. Cystoscopy and urine cytology were performed every 3 months within 2 years after surgery, every 6 months for the next 3 years, and then once a year. Abdominal computed tomography examinations were performed every 6 months after surgery for 2 years, and then once a year. All results were obtained through outpatient or telephone interviews. The period from the time of surgery to the last follow-up (February $\left.1^{\text {st }}, 2019\right)$ or the patient's death was recorded as the OS time period.

The study was in line with the Helsinki Declaration and approved by the Ethics Committee of the Tenth People's Hospital of Shanghai (SHSY-IEC-4.1/19-120/01). All participants provided written informed consent before registration.

\section{Statistical analysis}

SPSS v24.0 (SPSS Inc., Chicago, IL, USA) was used for all statistical analyses. Median and interquartile range values were used to evaluate continuous variables; frequency and scale were used to evaluate categorical variables. Chi- 
square test is used to analyze categorical variables. The optimal cut-off values for the SII, CAR, NLR, and PLR were obtained with $\mathrm{X}$-tile software (https://x-tile.software. informer.com/). Kaplan-Meier curves and log-rank tests were used to predict OS in relation to SII, CAR, NLR and PLR. Receiver operating characteristic (ROC) curves were generated and areas under the curves (AUCs) were determined. Univariate and multivariate Cox regression analyses were performed to calculate corresponding hazard ratios (HRs) and 95\% confidence interval (CI). Nomograms and calibration curves were created in $\mathrm{R}$ software (http://www.R-project.org). P values $<0.05$ were considered statistically significant.

\section{Results}

\section{Clinicopathological features of the patients}

In the primary cohort, there were 121 males and 18 females with a median age of 67 years (range, 29-87 years). Regarding TNM staging, there were 59 cases graded as Tis-T1, 32 cases graded as T2, 22 cases graded as T3, and 26 cases graded as T4. Forty-five patients died during follow-up. In the validation cohort, there were 61 males and 9 females with a median age of 66 years (range, 37-87 years). There were 23 cases graded as Tis-T1, 19 cases graded as T2, 17 cases graded as T3, and 11 cases graded as T4. Twentysix patients died during follow-up. Other clinicopathological parameters of the patients are summarized in Table 1. At the same time, there is no statistical difference between the two cohorts.

\section{Association of inflammatory biomarkers with clinicopathological features}

Optimal cutoff values for systemic inflammatory indexes determined in X-tile were as follows: SII (SII $\leq 507$, SII $>507)$, CAR (CAR $\leq 0.2$, CAR $>0.2)$, NLR $(\mathrm{NLR} \leq 2.3$, NLR >2.3), and PLR (PLR $\leq 132.5$, PLR >132.5) (Figure 1). Correlation values between systemic inflammatory indexes and clinicopathological features are shown in Table 2. In the primary cohort, we found that high SII levels were related to a larger tumor size, more advanced $\mathrm{T}$ stage, and poor lymph node status. These associations were replicated in the verification cohort. SII values correlated strongly with the NLR, PLR, and CAR inflammatory indexes in both cohorts (Table 2).

Stratified Kaplan-Meier OS curves showed that patients in the primary cohort with SII values $\leq 507$ had a longer OS than those with greater SII values (Figure $2 A$ ); at the same time, we divided the patients into $<\mathrm{T} 2 \mathrm{~N} 0 \mathrm{M} 0$, and $\geq \mathrm{T} 2 \mathrm{~N} 0 \mathrm{M} 0$ in the primary and validation cohort. We can see that the prognosis roles of SII seems to be greater in the $\geq \mathrm{T} 2 \mathrm{~N} 0 \mathrm{M} 0$ group; while, in the $<\mathrm{T} 2 \mathrm{~N} 0 \mathrm{M} 0$ group, because the survival time of the $\mathrm{T} 1$ stage patients is longer, the role of SII in evaluating prognosis is not as good as that of the $\geq \mathrm{T} 2 \mathrm{~N} 0 \mathrm{M} 0$ group (Figure $S 1)$. Similar results were obtained for CAR, NLR, and PLR (Figure 2B,C,D). ROC curve analysis showed that the AUC for the SII was larger than those for CAR, NLR, and PLR (Figure 2E); similar results were obtained in the validation cohort (Figure 3). Hence, SII appeared to be a better predictor of OS than the other inflammatory indexes analyzed.

\section{Univariate and multivariate analyses of $O S$}

Univariate analysis indicated that the SII, NLR, PLR, CAR, T stage, lymph node status, metastasis status, tumor size, tumor margin ,and vessel invasion were significantly related to OS in the primary cohort $(\mathrm{P}<0.05)$, whereas gender, age, $\mathrm{BMI}$, and surgical method had no significant influence on prognosis (Table 3). Multivariate analysis further indicated that SII, CAR, T stage, lymph node status, tumor size, and tumor margin were independent predictors of OS in the primary cohort of patients. In the validation cohort, we obtained similar results, with the SII, CAR, T stage, tumor size, and tumor margin being confirmed as independent prognostic factors.

\section{Development and validation of nomogram models}

An OS nomogram based on inflammatory actors and clinical pathological features was used to predict 3- and 5-year survival of the primary cohort of bladder cancer patients with a multivariate Cox regression analysis (Figure 4). Variable scores were based on the intersection of the vertical line and point axis associated with each variable, and total risk scores were summations of each variable score. These total scores established predictive measures of OS for each patient. The Harrell's C-index of the nomogram was 0.82. Analysis of time-dependent ROC curves for OS revealed that the AUC for the ROC curve (0.813) of the aforementioned nomogram was significantly larger than that of TNM stage (0.775), as shown in Figure 5A,B,C.

When the established nomogram was used to verify OS in the validation cohort, it was observed that the 3 - and 
Table 1 Clinicopathological characteristics of bladder cancer patients by cohort

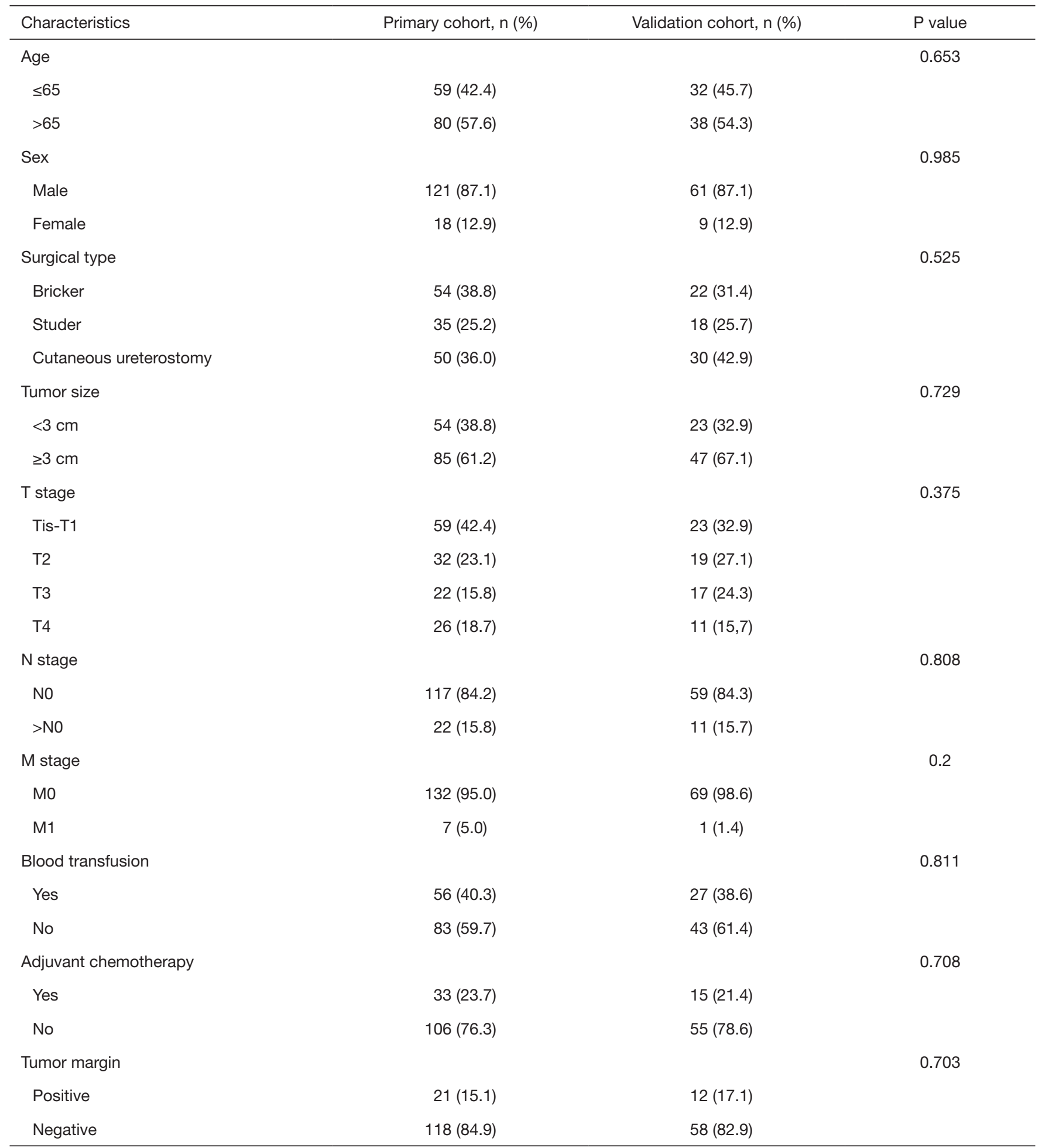

Table 1 (continued) 
Table 1 (continued)

\begin{tabular}{lcc}
\hline Characteristics & Primary cohort, $\mathrm{n}(\%)$ & Validation cohort, $\mathrm{n}(\%)$ \\
\hline Vessel invasion & $35(25.2)$ & $26(37.1)$ \\
Positive & $104(74.8)$ & $44(62.9)$ \\
Negative & & \\
BMl & $84(60.4)$ & $33(47.1)$ \\
$\leq 24$ & $55(39.6)$ & $37(52.9)$ \\
$>24$ & & \\
Diabetes & $24(17.3)$ & $11(15.7)$ \\
Yes & $115(82.7)$ & $59(84.3)$ \\
No & & \\
Cardiovascular disease & $57(41.0)$ & $26(37.1)$ \\
Yes & $82(59.0)$ & $44(62.9)$ \\
No & & 0.068 \\
\hline
\end{tabular}

BMI, body mass index.
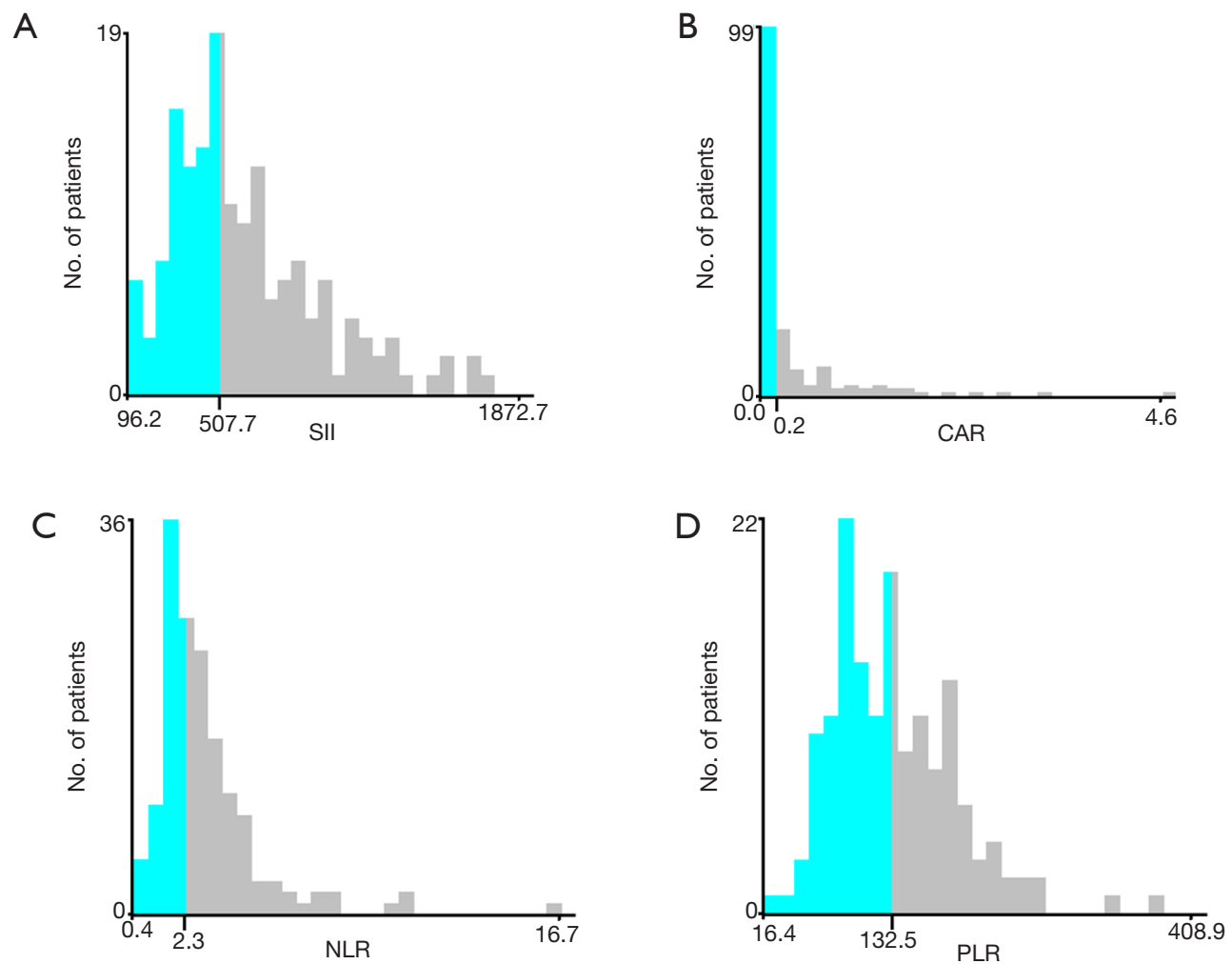

Figure 1 X-tile analyses of OS based on patient data to determine the optimal cut-off values for SII (A), CAR (B), NLR (C), and PLR (D). The optimal cut-off values are shown in histograms of the entire cohort. OS, overall survival; SII, systemic immune-inflammation index; NLR, neutrophil-lymphocyte ratio; PLR, platelet-lymphocyte ratio; CAR, C-reactive protein/albumin ratio. 
Page 6 of 14

Zhang et al. The role of SII in bladder cancer

Table 2 Correlation of SII with clinicopathologic characteristics by cohort

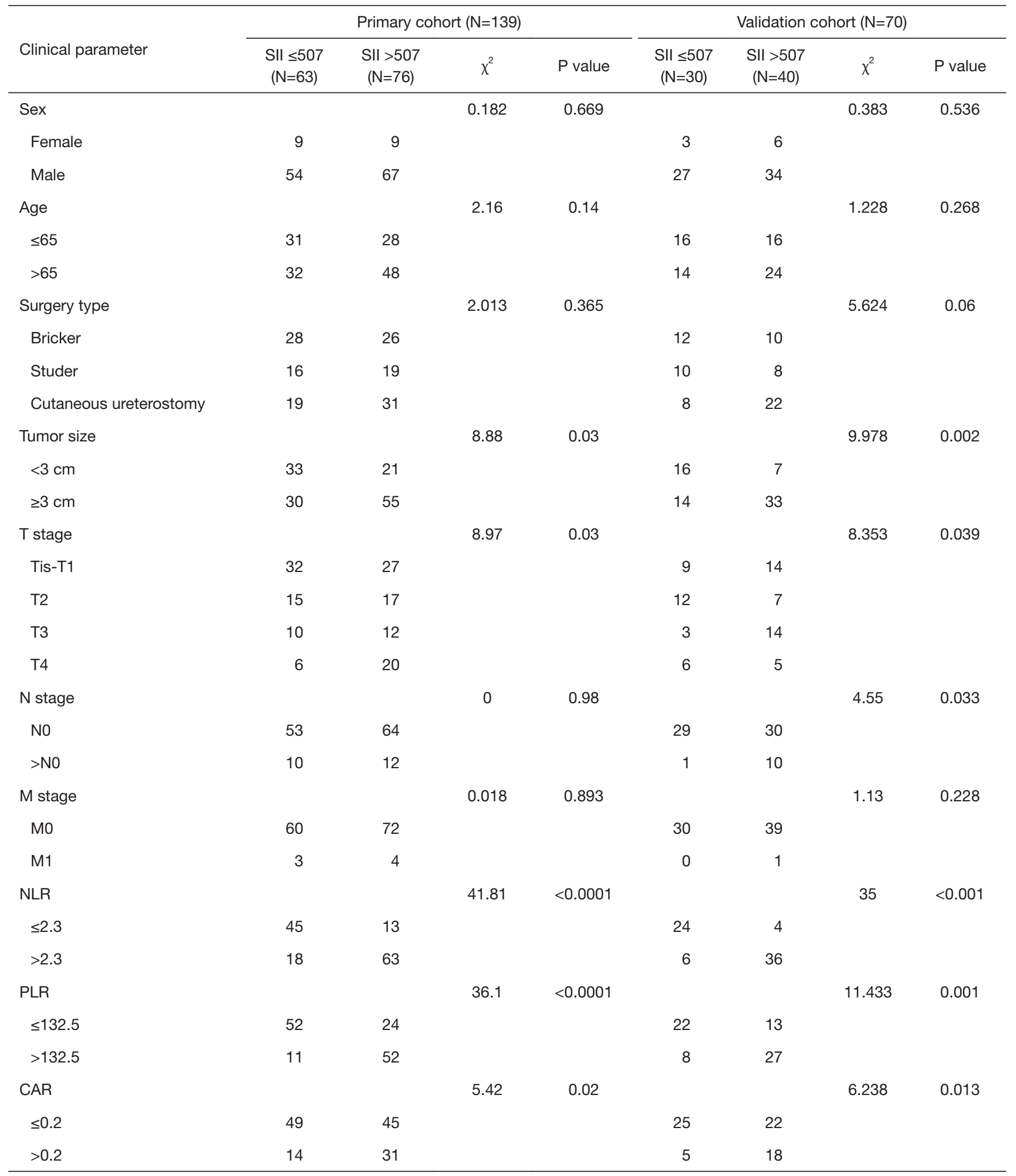

Table 2 (continued) 
Table 2 (continued)

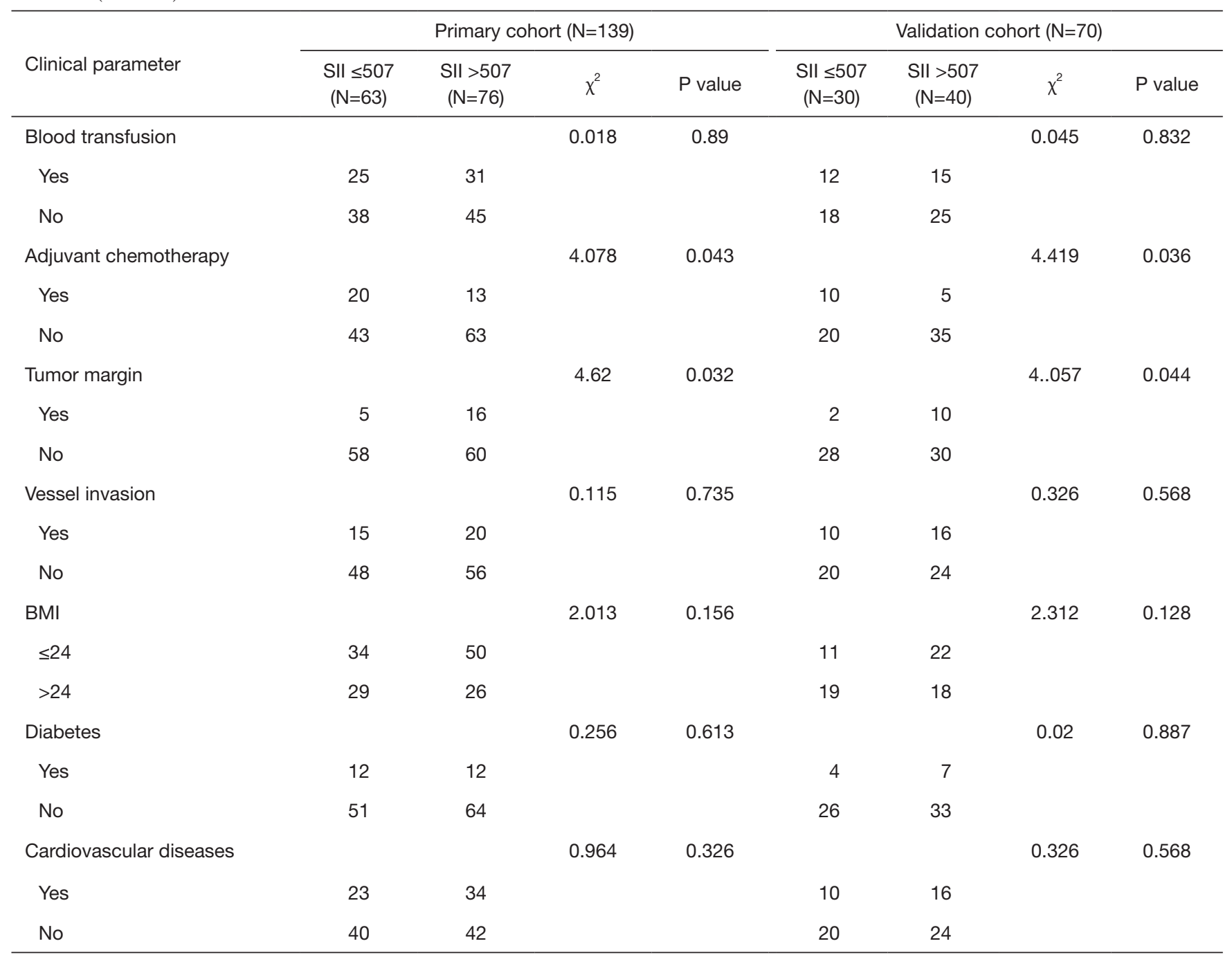

SII, systemic immune-inflammation index; NLR, neutrophil-lymphocyte ratio; PLR, platelet-lymphocyte ratio; CAR, C-reactive protein/ albumin ratio; BMI, body mass index.

5 -year OS rates predicted by the nomogram were consistent with actual observations. The C-index of the nomogram was 0.784 in the validation cohort. The AUC for the predictive nomogram ROC (0.852) in the validation cohort was also significantly greater than that for TNM stage (0.728), as shown in Figure 5D,E,F.

\section{Discussion}

The use of inflammation and immune response related markers have gained attention in recent years as potential predictors of the prognosis of cancer patients $(15,16)$. The emergent indicator SII has been shown to be related to prognosis for several cancers. This study systematically evaluated the role of SII in the prognosis of patients undergoing radical cystectomy. In this study, the SII was confirmed to be associated with tumor size, and tumor stage, and was found to be an independent prognostic factor in patients who have undergone radical cystectomy. The SII exhibited better predictive power than other existing prognostic indicators based on inflammatory parameters (i.e., NLR, PLR and CAR). The nomogram that we constructed based on biomarkers, namely the SII and clinical pathological parameters, provided accurate prediction of OS of postoperative bladder cancer patients, with an accuracy that was significantly higher than that 

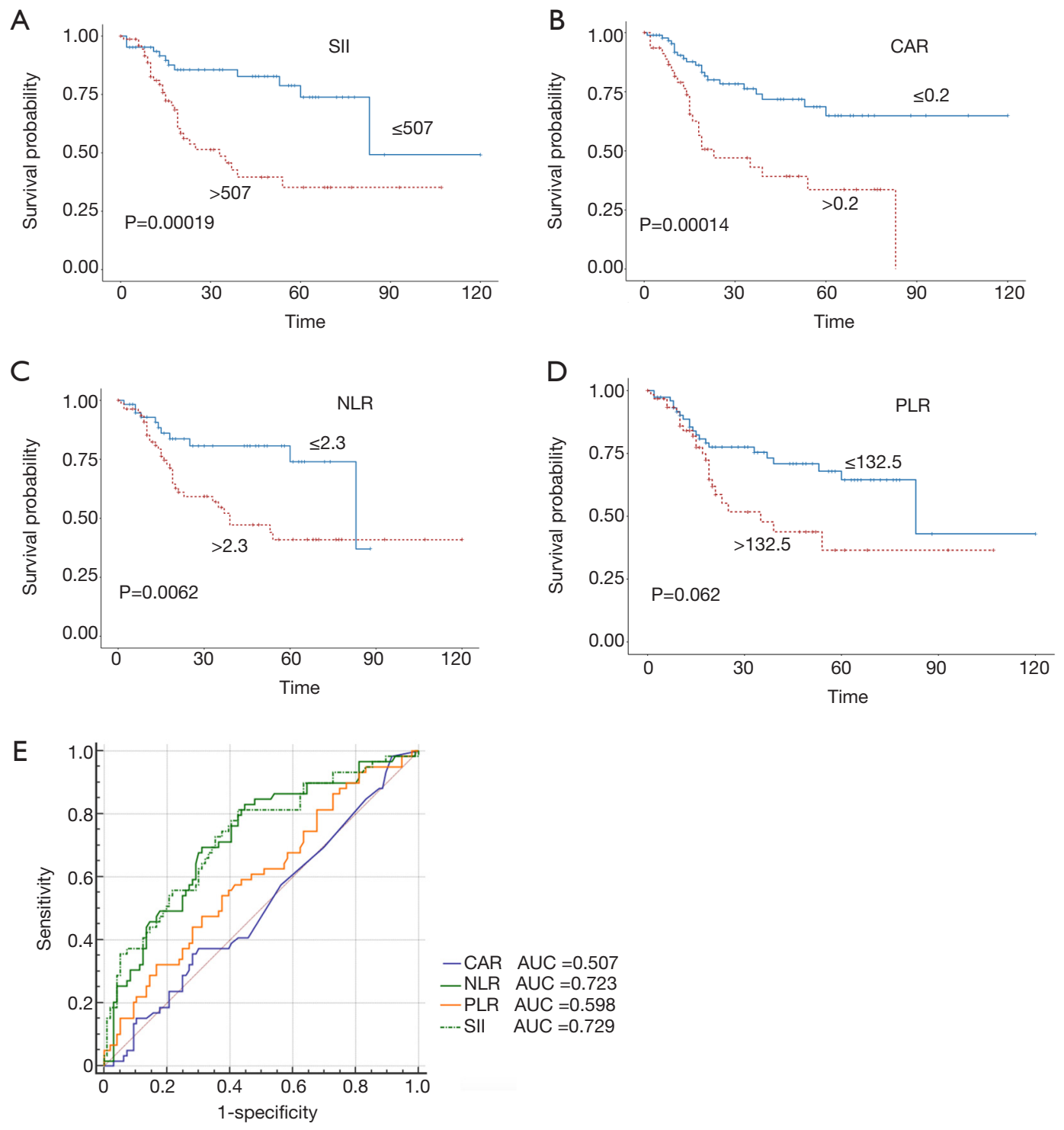

Figure 2 Predictive value of the SII (A), CAR (B), NLR (C), and PLR (D) cut-off values in bladder cancer prognosis in the primary cohort; (E) utility of the SII for predicting bladder cancer prognosis, compared with CAR, NLR, and PLR, as determined by ROC curve comparisons in the primary cohort. SII, systemic immune-inflammation index; NLR, neutrophil-lymphocyte ratio; PLR, platelet-lymphocyte ratio; CAR, C-reactive protein/albumin ratio; ROC, receiver operating characteristic; AUC, area under the curve.

obtained with TNM staging. Notwithstanding, we also noticed that there was no statistically significant difference between SII and bladder cancer grade, perhaps because most patients subjected to radical cystectomy in China have a high-grade pathological diagnosis.

Inflammation responses reflect systemic immune status and reveal the complexity of impaired immune surveillance caused by cancer cells (17). Previous studies have demonstrated associations of inflammation-related markers with bladder cancer patient prognosis. Notably, Our group found that preoperative CAR was a significant predictor of survival in bladder cancer patients after radical cystectomy (11). Additionally, Cheah and colleagues established that high CD14 expression in bladder cancer cells increased recruitment of inflammatory factors, polarizing monocytes and macrophages to alter their immunosuppressive characteristics and promote tumor cell growth (18). In the current study, we confirmed the 
A

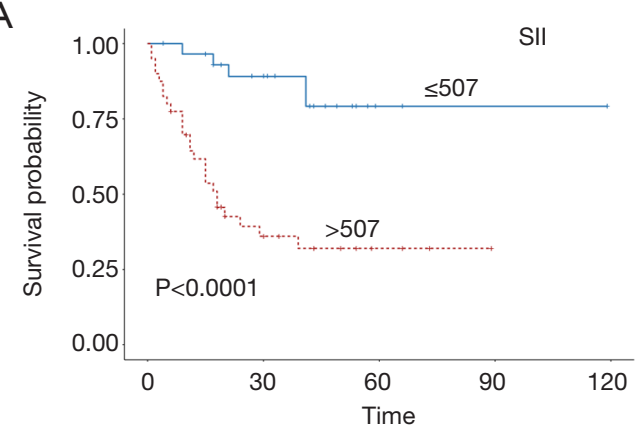

C
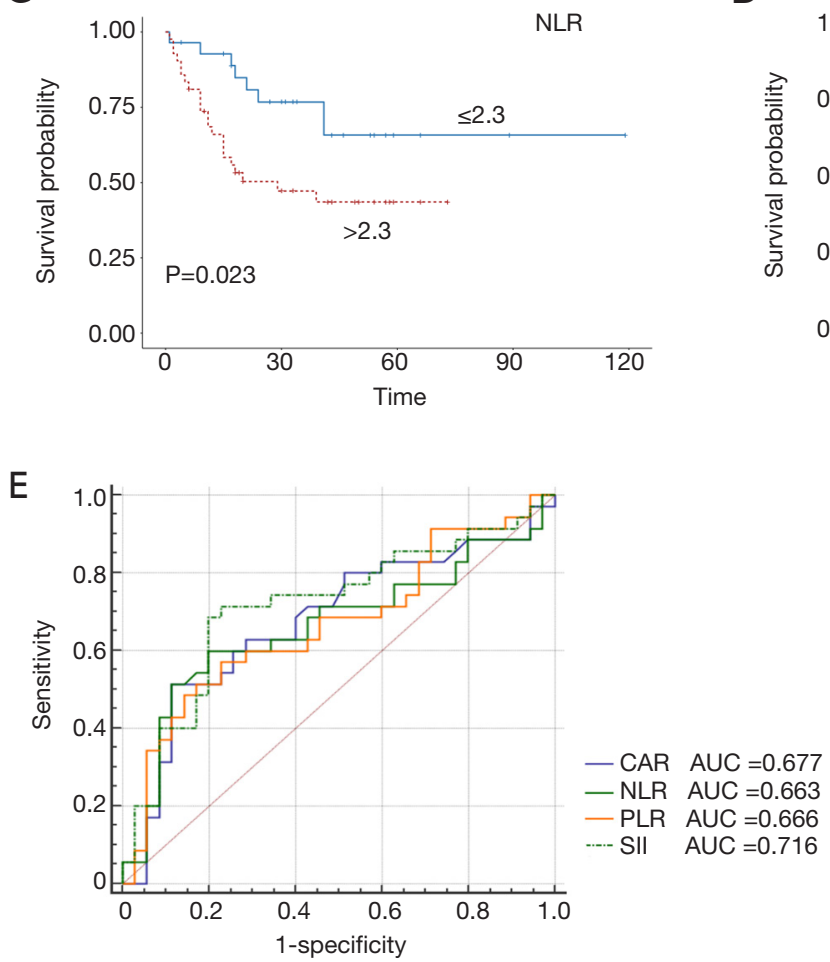

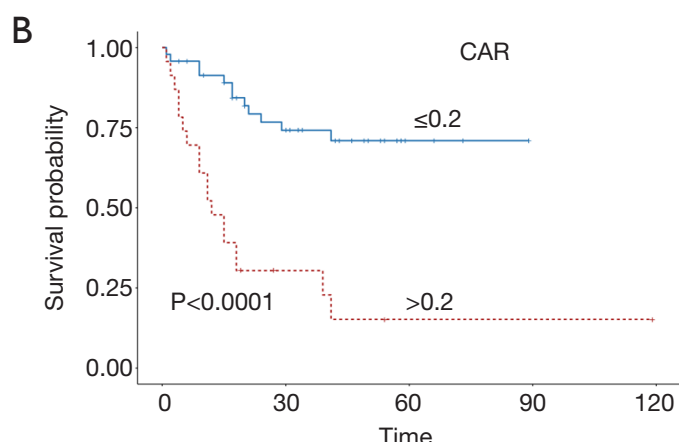

D

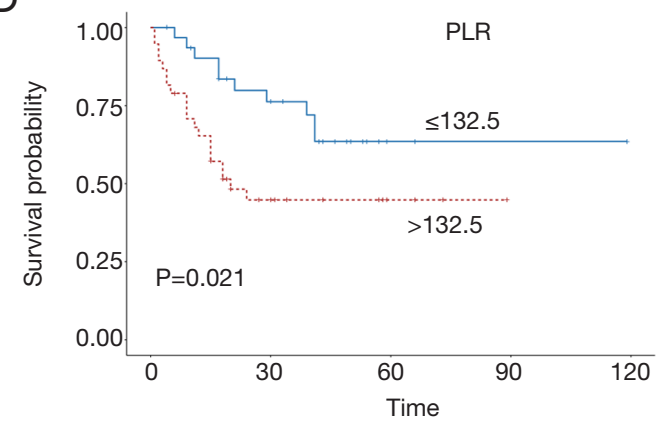

Figure 3 Predictive value of the SII (A), CAR (B), NLR (C), and PLR (D) cut-off values in bladder cancer prognosis in the validation cohort; (E) utility of the SII for predicting bladder cancer prognosis compared with CAR, NLR, and PLR, as determined by ROC curve comparisons in the validation cohort. SII, systemic immune-inflammation index; NLR, neutrophil-lymphocyte ratio; PLR, plateletlymphocyte ratio; CAR, C-reactive protein/albumin ratio; ROC, receiver operating characteristic; AUC, area under the curve.

association of the SII with bladder cancer as an independent prognostic predictor

Neutrophils can release inflammatory factors involved in tumor proliferation and metastasis, such as neutrophil elastase, matrix metalloproteinase-9, and interleukin-8. NLR reflects immune responses related to cancer immunosurveillance, immune-editing, and immunesubversion (19). More specifically, an increased NLR indicates an increase in neutrophils, relative to lymphocytes, leading to the promotion of tumor inflammation and increasing cancer metastasis and recurrence risks (20). Meanwhile, neutrophils secrete interleukin-6, vascular endothelial growth factor, and transforming growth factor beta in the tumor microenvironment, resulting in an induction of lymphocyte apoptosis and immunosuppression (21).

Observations indicating that platelets play an important role in the recruitment of monocytes and granulocytes into neoplasms (17) suggest that platelet functionality may be 
Table 3 Univariate and multivariate Cox regression analyses for OS in patients with bladder cancer

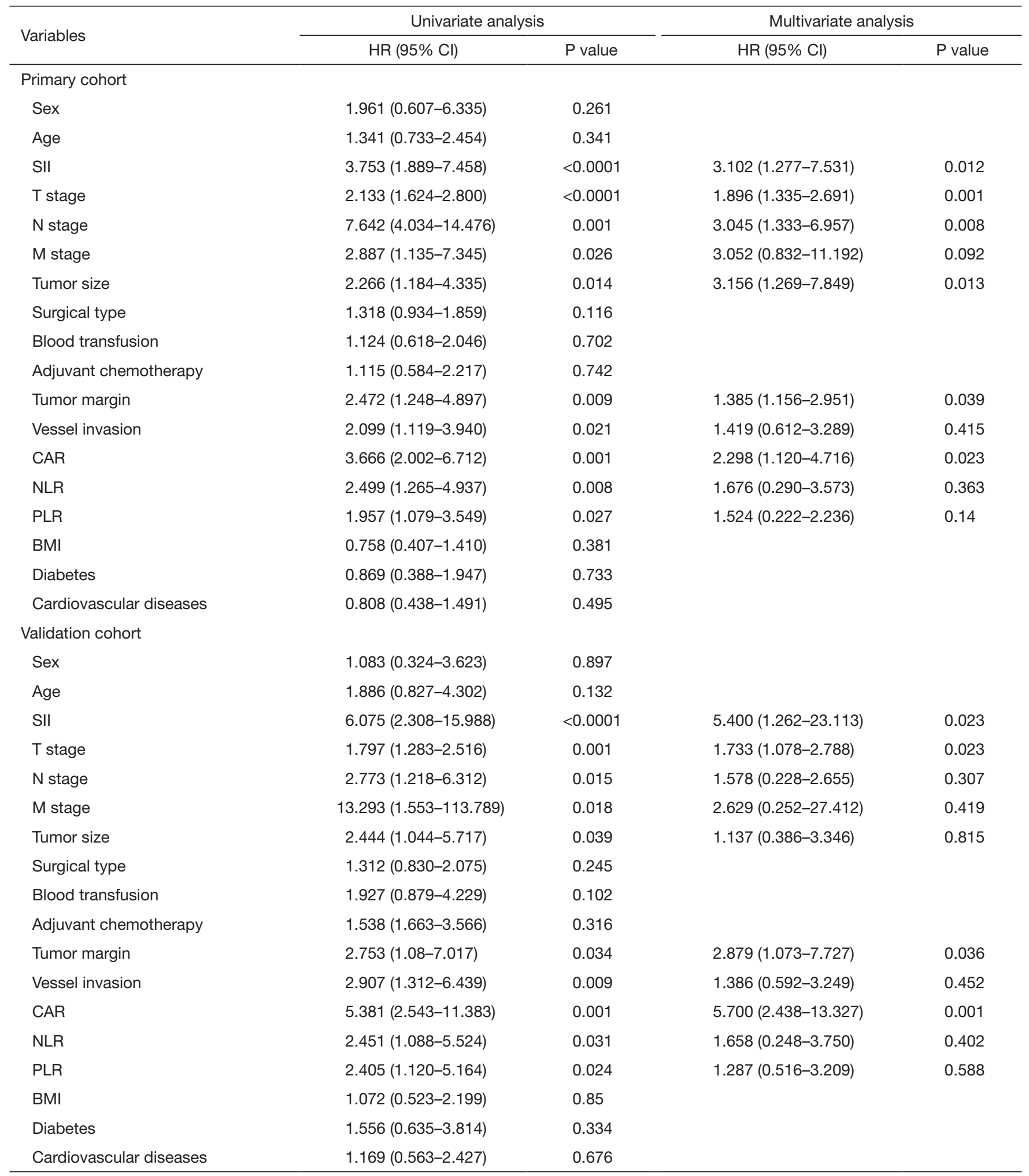

OS, overall survival; HR, hazard ratio; $\mathrm{Cl}$, confidence interval; SII, systemic immune-inflammation index; NLR, neutrophil-lymphocyte ratio; PLR, platelet-lymphocyte ratio; CAR, C-reactive protein/albumin ratio; BMI, body mass index. 


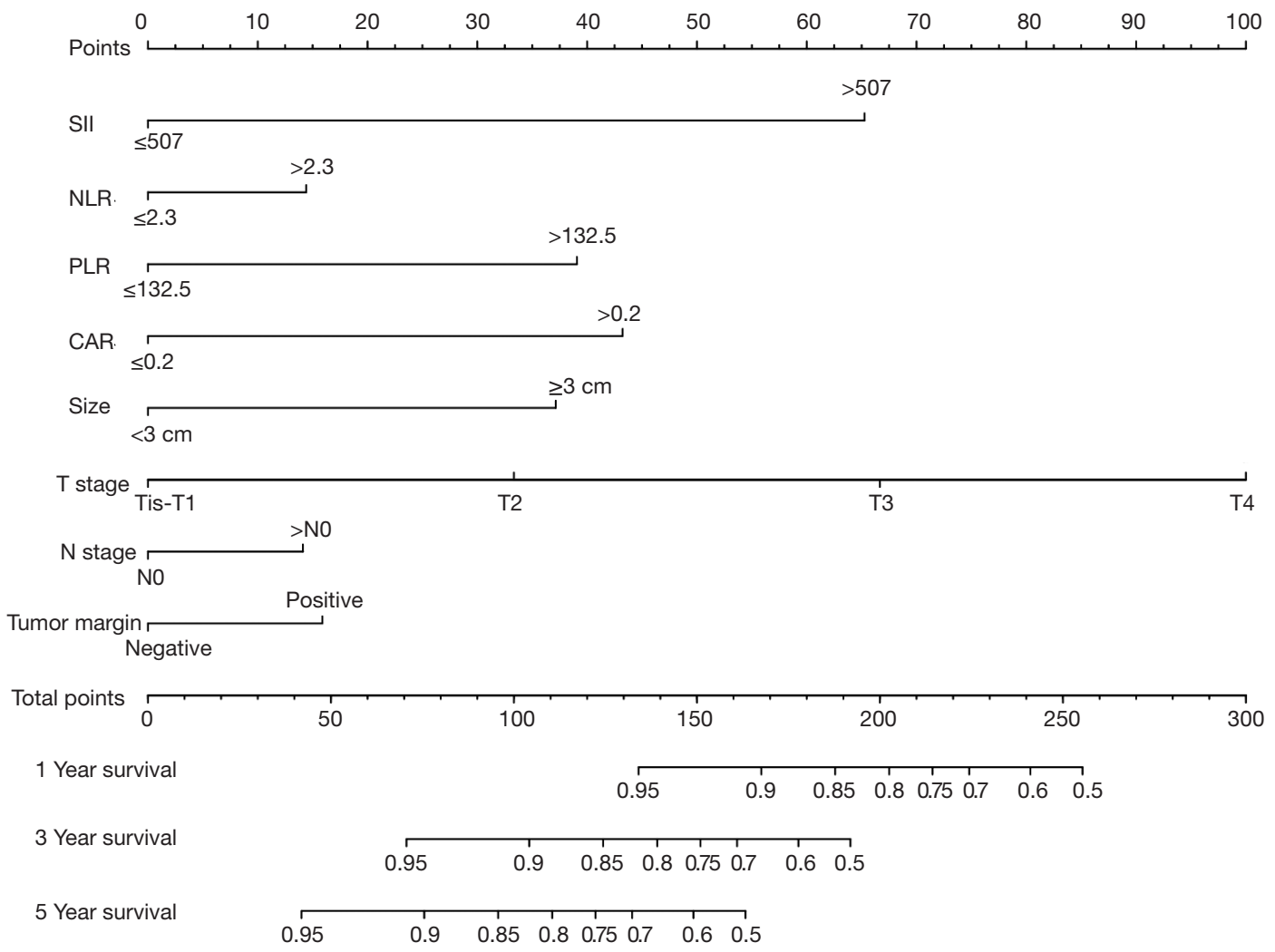

Figure 4 Nomogram developed by integrating multiple immune and inflammation indexes and clinical pathological parameters in patients with bladder cancer. SII, systemic immune-inflammation index; NLR, neutrophil-lymphocyte ratio; PLR, platelet-lymphocyte ratio; CAR, C-reactive protein/albumin ratio.

critical for the production of tumor-associated macrophages and neutrophils (22). Platelets can also stimulate tumor cells directly, which may promote tumor growth, invasion, and angiogenesis (23).

Following antigen stimulation, lymphocytes generate important cellular components of the immune response. They secrete cytokines (e.g., interferon- $\gamma$ and tumor necrosis factor- $\alpha$ ) that trigger specific immune responses, thereby controlling tumor growth and improving the prognosis of cancer patients (24). Low lymphocyte numbers or function is associated with weakened immune surveillance and activity (25). The SII is a systemic inflammatory index based on neutrophil, platelet, and lymphocyte levels that may be used to monitor bodily immune status.

Recently, nomograms have been used to predict the probability of outcomes in a variety of cancer patients. Xu et al. constructed a nomogram to predict the recurrence of bladder cancer where 71 patients were divided into training $(\mathrm{N}=50)$ and validation cohorts $(\mathrm{N}=21)$. Magnetic resonance imaging (MRI) parameters and clinical risk factors (tumor stage, operation styles, etc.) were included in the nomogram. The study showed the bladder cancer recurrence within 2 years can be predicted using nomogram. However, this study included only few clinical pathological parameters (26). In our study, we added inflammation and immune status as well as more clinical pathological data for better result. At the same time, we were attentive to the patient's OS, with the aim of better personalizing treatment plans for patients. Interestingly, Riester et al. were able to improve the accuracy of their nomogram by including gene expression information (27). However, the inclusion of such data is associated with some increase in cost. Thus, inclusion of inflammation indicators in the SII has the advantage of being relatively inexpensive and thus more applicable across different settings, including in countries with developing economies, such as China.

Limitations of this research include the study being a single-center retrospective cohort. Thus, the primary 
A Primary cohort

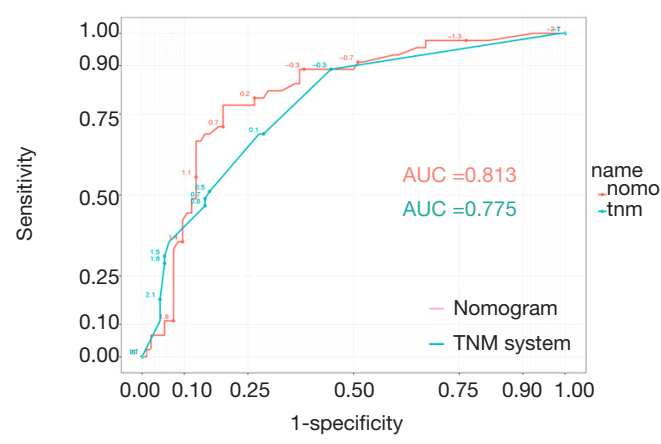

B

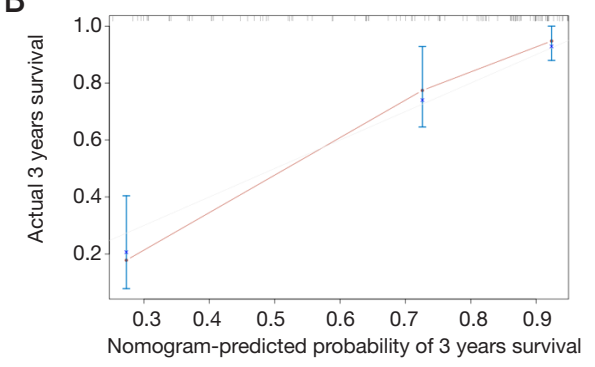

C

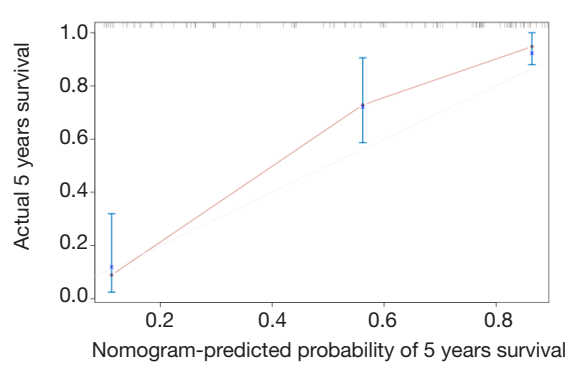

D Validation cohort

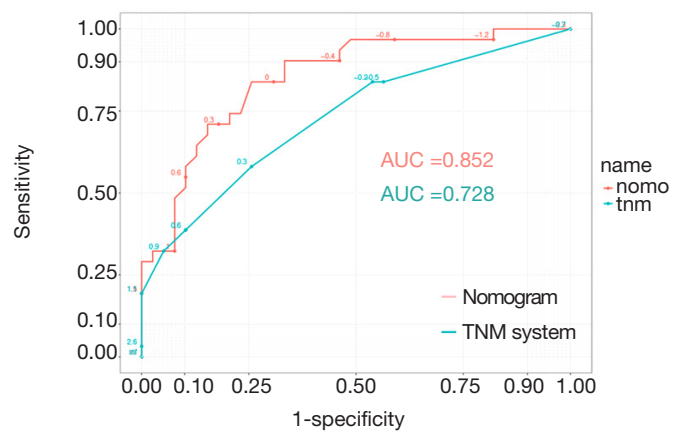

E

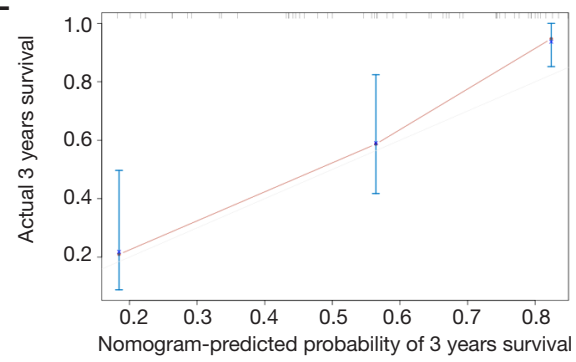

F

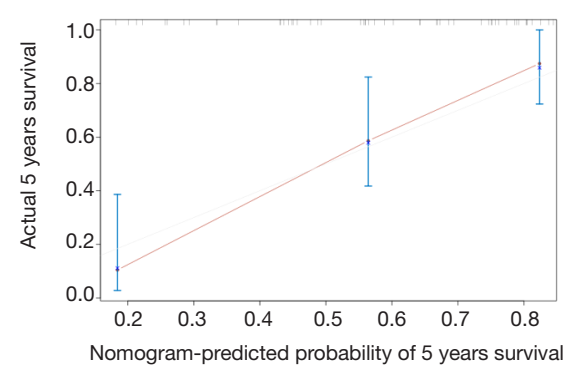

Figure 5 Time-dependent ROC curves by nomogram and TNM staging in the primary cohort (A); calibration curved for predicting patient survival at 3 years (B) and 5 years $(\mathrm{C})$ in the primary cohort; time-dependent ROC curves by nomogram and TNM staging in the validation cohort (D); calibration curves for predicting patient survival at 3 years (E) and 5 years $(\mathrm{F})$ in the validation cohort. ROC, receiver operating characteristic; TNM, tumor, lymph node, metastasis; AUC, area under the curve.

and validation cohort datasets were from the same center, which may affect the generalizability of the findings across diverse populations. Furthermore, the data for this group of patients may not represent other bladder cancer patients who refused surgical intervention for various reasons. In addition, this study was based on a retrospective analysis that examined the relationship between immune inflammatory markers and OS. Further work is needed to examine the relationship of these indicators with tumor recurrence and tumor-specific survival. Thus, in the future, we hope to conduct a multicenter and forward-looking cohort study.

\section{Conclusions}

In conclusion, the present data suggest that the SII can be used as an independent predictor of prognosis in bladder cancer patients after radical cystectomy. The nomogram presented here, which is based on inflammatory-immune factors, can be to guide individualized treatment planning. The ease of use and low cost of the nomogram make it appropriate for a great variety of clinical settings, including 
in developing countries such as China.

\section{Acknowledgments}

The authors thank all those who participated in recruitment of patients and helping to gather patients' information. Funding: This work was supported in part by a grant from the National Natural Science Foundation of China (\#81472389); Shanghai Science Committee Foundation (\#19411967700).

\section{Footnote}

Conflicts of Interest: The authors have no conflicts of interest to declare.

Ethical Statement: The authors are accountable for all aspects of the work in ensuring that questions related to the accuracy or integrity of any part of the work are appropriately investigated and resolved. The study was in line with the Helsinki Declaration and approved by the Ethics Committee at the Tenth People's Hospital of Shanghai (SHSY-IEC-4.1/19-120/01). The study outcomes will not affect the future management of the patients. The use of human blood samples was in accordance with the legislation in China. Informed consent was obtained from the controls and patients or their relatives.

\section{References}

1. Siegel RL, Miller KD, Jemal A. Cancer statistics, 2016. CA Cancer J Clin 2016;66:7-30.

2. Chen $W$, Zheng R, Baade PD, et al. Cancer statistics in China, 2015. CA Cancer J Clin 2016;66:115-32.

3. Cumberbatch MGK, Jubber I, Black PC, et al. Epidemiology of bladder cancer: a systematic review and contemporary update of risk factors in 2018. Eur Urol 2018;74:784-95.

4. Marcos-Gragera R, Mallone S, Kiemeney LA, et al. Urinary tract cancer survival in Europe 1999-2007: results of the population-based study EUROCARE-5. Eur J Cancer 2015;51:2217-30.

5. Babjuk M, Böhle A, Burger M, et al. EAU guidelines on non-muscle-invasive urothelial carcinoma of the bladder: update 2016. Eur Urol 2017;71:447-61.

6. Martin-Doyle W, Leow JJ, Orsola A, et al. Improving selection criteria for early cystectomy in high-grade t1 bladder cancer: a meta-analysis of 15,215 patients. J Clin
Oncol 2015;33:643-50.

7. Gilbert SM, Wood DP, Dunn RL, et al. Measuring health-related quality of life outcomes in bladder cancer patients using the Bladder Cancer Index (BCI). Cancer 2007;109:1756-62.

8. Hanahan D, Weinberg RA. Hallmarks of cancer: the next generation. Cell 2011;144:646-74.

9. Marchioni M, Primiceri G, Ingrosso M, et al. The clinical use of the neutrophil to lymphocyte ratio (NLR) in urothelial cancer: a systematic review. Clin Genitourin Cancer 2016;14:473-84.

10. Kaynar M, Yıldırım ME, Badem H, et al. Bladder cancer invasion predictability based on preoperative neutrophillymphocyte ratio. Tumour Biol 2014;35:6601-5.

11. Guo Y, Cai K, Mao S, et al. Preoperative C-reactive protein/albumin ratio is a significant predictor of survival in bladder cancer patients after radical cystectomy: a retrospective study. Cancer Manag Res 2018;10:4789-804.

12. Shi H, Jiang Y, Cao H, et al. Nomogram based on systemic immune-inflammation index to predict overall survival in gastric cancer patients. Dis Markers 2018;2018:1787424.

13. Xie QK, Chen P, Hu WM, et al. The systemic immuneinflammation index is an independent predictor of survival for metastatic colorectal cancer and its association with the lymphocytic response to the tumor. J Transl Med 2018; 16:273.

14. Geng Y, Shao Y, Zhu D, et al. Systemic immuneinflammation index predicts prognosis of patients with esophageal squamous cell carcinoma: a propensity scorematched analysis. Sci Rep 2016;6:39482.

15. Ryan AM, Power DG, Daly L, et al. Cancer-associated malnutrition, cachexia and sarcopenia: the skeleton in the hospital closet 40 years later. Proc Nutr Soc 2016;75:199-211.

16. Eftekhari R, de Lima SG, Liu Y, et al. Microenvironment proteinases, proteinase-activated receptor regulation, cancer and inflammation. Biol Chem 2018;399:1023-39.

17. Kim J, Bae JS. Tumor-associated macrophages and neutrophils in tumor microenvironment. Mediators Inflamm 2016;2016:6058147.

18. Cheah MT, Chen JY, Sahoo D, et al. CD14-expressing cancer cells establish the inflammatory and proliferative tumor microenvironment in bladder cancer. Proc Natl Acad Sci U S A 2015;112:4725-30.

19. Block MS, Markovic SN. The tumor/immune interface: clinical evidence of cancer immunosurveillance, immunoediting and immunosubversion. Am J Immunol 2009;5:29-49. 
20. Capone M, Giannarelli D, Mallardo D, et al. Baseline neutrophil-to-lymphocyte ratio (NLR) and derived NLR could predict overall survival in patients with advanced melanoma treated with nivolumab. J Immunother Cancer 2018;6:74.

21. Maeda K, Malykhin A, Teague-Weber BN, et al. Interleukin-6 aborts lymphopoiesis and elevates production of myeloid cells in systemic lupus erythematosus-prone B6.Sle1.Yaa animals. Blood 2009;113:4534-40.

22. Gros A, Ollivier V, Ho-Tin-Noé B. Platelets in inflammation: regulation of leukocyte activities and vascular repair. Front Immunol 2015;5:678.

23. Franco AT, Corken A, Ware J. Platelets at the interface of thrombosis, inflammation, and cancer. Blood

Cite this article as: Zhang $\mathrm{W}$, Wang $\mathrm{R}, \mathrm{Ma} \mathrm{W}, \mathrm{Wu} \mathrm{Y}$, Maskey N, Guo Y, Liu J, Mao S, Zhang J, Yao X, Liu Y. Systemic immune-inflammation index predicts prognosis of bladder cancer patients after radical cystectomy. Ann Transl Med 2019;7(18):431. doi: 10.21037/atm.2019.09.02
2015;126:582-8.

24. Ohtani H. Focus on TILs: prognostic significance of tumor infiltrating lymphocytes in human bladder cancer. Cancer Immun 2007;7:4.

25. Altman BJ, Dang CV. Normal and cancer cell metabolism: lymphocytes and lymphoma. FEBS J 2012;279:2598-609.

26. Xu X, Wang H, Du P, et al. A predictive nomogram for individualized recurrence stratification of bladder cancer using multiparametric MRI and clinical risk factors. J Magn Reson Imaging 2019. [Epub ahead of print].

27. Riester M, Taylor JM, Feifer A, et al. Combination of a novel gene expression signature with a clinical nomogram improves the prediction of survival in high-risk bladder cancer. Clin Cancer Res 2012;18:1323-33. 


\section{Supplementary}

A Primary cohort
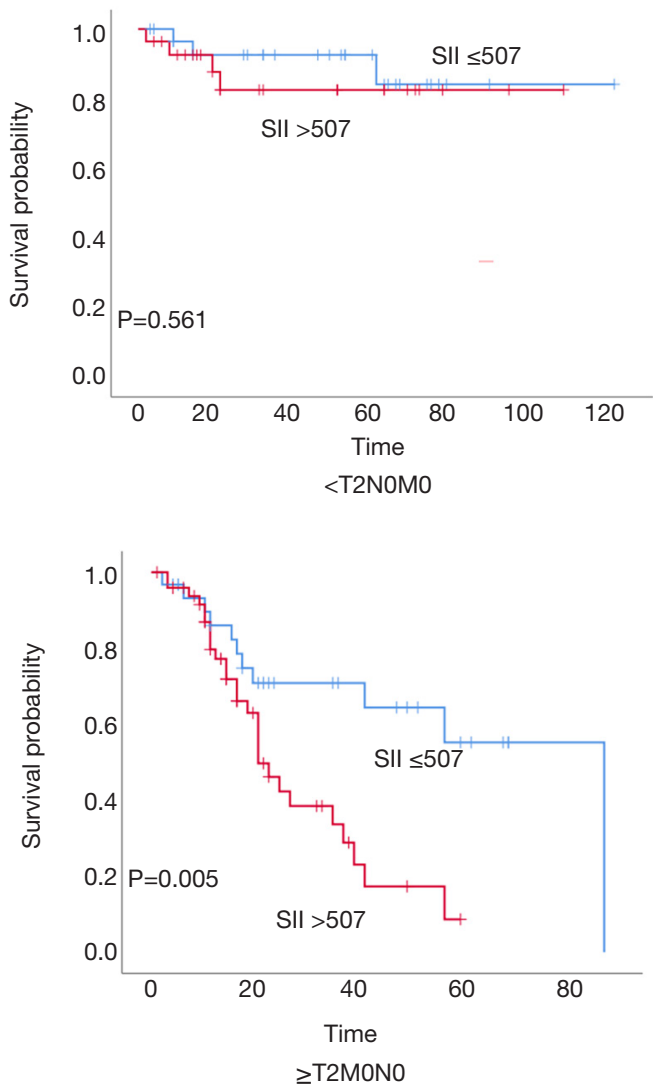
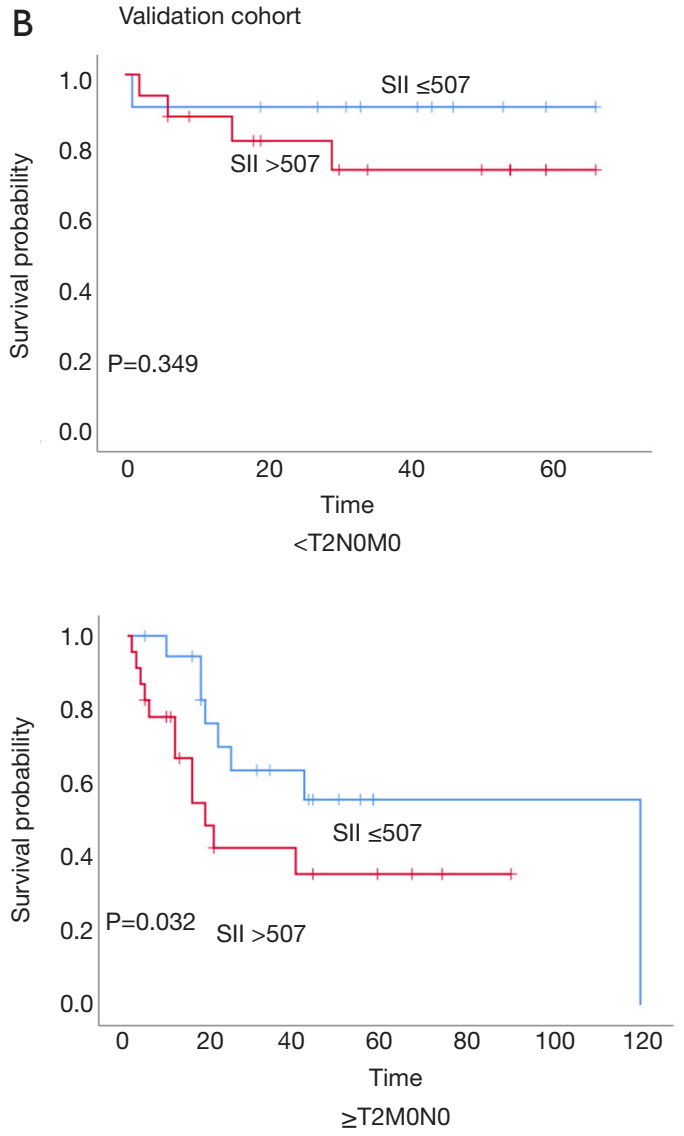

Figure S1 Prognostic roles of SII for different T stages in primary and validation cohorts. (A) In primary cohort, the prognosis roles of SII would be greater in the $\geq \mathrm{T} 2 \mathrm{~N} 0 \mathrm{M} 0$ group; (B) in validation cohort, the prognosis roles of SII would be greater in the $\geq \mathrm{T} 2 \mathrm{~N} 0 \mathrm{M} 0$ group. SII, systemic immune-inflammation index. 